\title{
Fast head-tail instability with space charge
}

\author{
M. Blaskiewicz \\ Alternating Gradient Synchrotron Department, Brookhaven National Laboratory, Upton, New York 11973-5000
}

(Received 29 April 1998; published 13 August 1998)

\begin{abstract}
The fast head-tail instability with space charge is studied using series expansion techniques, numerical simulations, and a new formulation which allows for precise estimates of growth rates and thresholds. In regimes where they are reliable, all three techniques predict that space charge suppresses the fast head-tail instability. It is found that the series expansion techniques are unreliable for parameter regimes commonly employed in hadron accelerators. The numerical techniques are less prone to error, but the computational requirements become severe as space charge tune shifts increase. The new model has neither of these problems, but it underestimates the benefits of chromaticity, at least in its simplest form. [S1098-4402(98)00010-X]

PACS numbers: 29.27
\end{abstract}

\section{INTRODUCTION}

The fast head-tail or transverse mode coupling instability can limit the intensity of electron synchrotrons. In electron machines, the transverse impedance is usually dominated by features in the vacuum chamber such as kickers, rf cavities, and bellows. As a rule of thumb, the fast head-tail instability appears when the betatron tune shift becomes comparable to the synchrotron tune. Detailed studies have been performed and fast headtail thresholds have been used to constrain the machine impedance [1]. In low and medium energy hadron synchrotrons, the transverse impedance is usually dominated by incoherent space charge, which derives from mutual electrostatic repulsion of the particles within the beam. The transverse impedance of the vacuum chamber is usually much smaller, but still significant. To the author's knowledge, the fast head-tail instability has never been observed in a hadron machine. The purpose of this study is to explain why this instability is absent and to give machine designers techniques for ascertaining its importance in future accelerators.

Space charge alone does not appear to cause transverse instabilities [2], but the effect of space charge in conjunction with other sources of transverse impedance is less clear [3]. One of the main difficulties lies in the fact that the space charge forces often lead to betatron tune shifts $\Delta Q_{s c}$ that are large compared to the synchrotron tune $Q_{s}$. For example, in the Brookhaven alternating-gradient synchrotron (AGS) at injection $\Delta Q_{s c} / Q_{s} \approx 125$, while $\Delta Q_{s c} / Q_{s} \gtrsim 20$ is expected for the relativistic heavy ion collider (RHIC). In this regime, the simple tune shift formulas [4] are inappropriate, since their derivation assumes that the betatron tune shift is small compared to the synchrotron tune. This paper considers the regime where $\Delta Q_{s c} / Q_{s}$ can be large and where the synchrotron tune is small enough to neglect synchrobetatron resonances.

In Sec. II, the physical model is introduced. The linearized Vlasov equation for the system is derived and a solution is attempted using the basis expansion formalism
$[2,3,5,6]$. Results for boxcar and Gaussian line densities are presented. The solutions are not monotonic in $\Delta Q_{s c}$ and in some cases do not converge as more basis vectors are included. Additionally, the $Q_{s}=0$ limit does not agree with an exact calculation.

Section III contains a tracking algorithm employing discretized versions of the equations of motion. A fast algorithm which retains the symplecticity of the space charge force is derived [7]. Dependencies on the various, purely numerical, parameters are explored and regimes where their effects seem negligible are found.

In Sec. IV, the parabolic longitudinal potential is approximated as a square well, an approximation previously used for longitudinal instabilities [8,9]. For a longitudinal distribution with a well-defined synchrotron tune, this approximation yields an eigenvalue problem which is easily solved to machine precision. It is found that this model agrees with tracking in a parabolic longitudinal potential for zero chromaticity.

The conclusions are summarized in Sec. V.

\section{PHYSICAL MODEL}

For the purposes of beam dynamics, one transverse and the longitudinal degrees of freedom are considered. Let $\theta$ denote the machine azimuth, which increases by $2 \pi$ each turn, and will be used as the timelike variable. The time is denoted by $t$, and $\omega_{0}$ is the angular revolution frequency of a synchronous particle. Consider a single particle and let $\tau(\theta)=\omega_{0} t-\theta$ denote the time delay between this particle reaching $\theta$ and the synchronous particle reaching $\theta$ measured in radians of azimuth. The longitudinal equation of motion for a single particle is approximated as

$$
\frac{d^{2} \tau}{d^{2} \theta}+Q_{s}^{2} \tau=0
$$

where $Q_{s}$ is the synchrotron tune. The single particle equation of motion for the transverse degree of freedom $x$ 
is approximated as

$$
\begin{aligned}
\frac{d^{2} x}{d^{2} \theta}+Q_{x}^{2} x= & C_{s c} \rho(\theta, \tau)[x-\langle x(\theta, \tau)\rangle] \\
& +\int_{-T}^{\tau} d \tau^{\prime} W\left(\tau-\tau^{\prime}\right) \rho\left(\theta, \tau^{\prime}\right) \\
& \times\left\langle x\left(\theta, \tau^{\prime}\right)\right\rangle d \tau^{\prime}
\end{aligned}
$$

In Eq. (2), $Q_{x}$ is the bare betatron tune, $C_{s c} \geq 0$ characterizes the peak strength of the incoherent space charge force, and $\rho(\theta, \tau)$ is the line density of the particles, which vanishes for $|\tau|>T$, the half length of the bunch. The transverse center of the beam as a function of azimuth and delay is $\langle x(\theta, \tau)\rangle$, and the causal coherent forces due to wall impedances are characterized by the wake potential $W(\tau)$.

The solutions of Eqs. (1) and (2) form the basis of the paper. The first equation is trivial. It states that the longitudinal synchrotron oscillations have a single frequency and are unaffected by the transverse dynamics. This is an approximation, since path length depends on betatron amplitude. Amplitude dependence of the synchrotron frequency is also neglected. Equation (2) is more complicated. The left-hand side describes betatron oscillations in the smooth approximation with zero chromaticity, while the collective forces are on the right-hand side. The first term on the right-hand side approximates the incoherent space charge force, which is proportional to the local longitudinal density $\rho(\theta, \tau)$. The space charge force depends on the difference between the particle's transverse position $x$ and the average position for all particles having the same delay at that azimuth $\langle x(\theta, \tau)\rangle$. Variations in transverse beam size are neglected. It is known that this approximation is not adequate for describing quadrupolar breathing modes [10] in that the maximum space charge tune shift for a uniform density beam interacting with a quadrupole stopband is about 4/3 times that predicted using Eq. (2). However, to lowest order, transverse instabilities involve transverse dipole modes for which the approximation appears adequate. The second term on the right-hand side of Eq. (2) approximates the broadband wake force. The integral is only over the bunch, so the cumulative effects of previous turns are neglected. This approximation, as the assumption of zero chromaticity, is not needed and will be removed in Sec. IV.

To proceed analytically define the conjugate variables $p \equiv d x / d \theta, v \equiv d \tau /\left(Q_{s} d \theta\right)$ and consider the smooth phase space density $f(x, p, \tau, v, \theta)$ with $f d x d p d \tau d v$ being the number of particles in the phase space element $d x d p d \tau d v$ at azimuth $\theta$. From this phase space density define the three moments

$$
\begin{gathered}
\Pi(\tau, v, \theta)=\int d x \int d p f(x, p, \tau, v, \theta), \\
D(\tau, v, \theta)=\int x d x \int d p f(x, p, \tau, v, \theta), \\
P(\tau, v, \theta)=\int d x \int p d p f(x, p, \tau, v, \theta) .
\end{gathered}
$$

With these and previous definitions, the equations of motion, and no further approximations, it follows that [11]

$$
\begin{gathered}
\frac{d \Pi(\tau, v, \theta)}{d \theta}=0 \\
\frac{d D(\tau, v, \theta)}{d \theta}=P(\tau, v, \theta) \\
\frac{d P(\tau, v, \theta)}{d \theta}=-Q_{x}^{2} D(\tau, v, \theta)+\Pi(\tau, v, \theta) \\
\times \int_{-T}^{\tau} W\left(\tau-\tau^{\prime}\right) d \tau^{\prime} \int_{-\infty}^{\infty} d v^{\prime} D\left(\tau^{\prime}, v^{\prime}, \theta\right) \\
-C_{s c} \Pi(\tau, v, \theta) \int_{-\infty}^{\infty} d v^{\prime} D\left(\tau, v^{\prime}, \theta\right) \\
+C_{s c} D(\tau, v, \theta) \int_{-\infty}^{\infty} d v^{\prime} \Pi\left(\tau, v^{\prime}, \theta\right)
\end{gathered}
$$

where $d / d \theta \equiv \partial / \partial \theta+Q_{s}(v \partial / \partial \tau-\tau \partial / \partial v)$ is the total derivative operator.

Assume that the beam is matched longitudinally with $\Pi(\tau, v, \theta)=\Psi\left(v^{2}+\tau^{2}\right)$. This solves Eq. (6) and makes Eqs. (7) and (8) linear in the unknown functions. At this point, another approximation is made. Suppose $D(\tau, v, \theta)=\hat{D}(\tau, v, \theta) \exp \left(-i Q_{x} \theta\right)$ and $P(\tau, v, \theta)=\hat{P}(\tau, v, \theta) \exp \left(-i Q_{x} \theta\right)$. Insert these expressions into Eqs. (7) and (8) and make the approximation $d^{2} D / d^{2} \theta \approx-Q_{x}^{2} D-2 i Q_{x} \exp \left(-i Q_{x} \theta\right) d \hat{D} / d \theta$. This is equivalent to assuming that all of the tune shifts are small compared to $Q_{x}$ and restricting the calculation to the upper betatron sideband. The resulting equation for $\hat{D}$ is

$$
\begin{aligned}
\frac{\partial \hat{D}(\tau, v, \theta)}{\partial \theta}+Q_{s}\left\{v \frac{\partial \hat{D}(\tau, v, \theta)}{\partial \tau}-\tau \frac{\partial \hat{D}(\tau, v, \theta)}{\partial v}\right\}= & \frac{i}{2 Q_{x}} \Psi\left(v^{2}+\tau^{2}\right) \int_{-T}^{\tau} d \tau^{\prime} W\left(\tau-\tau^{\prime}\right) \int_{-\infty}^{\infty} d v^{\prime} \hat{D}\left(\tau^{\prime}, v^{\prime}, \theta\right) \\
& +\frac{i C_{s c}}{2 Q_{x}}\left\{\hat{D}(\tau, v, \theta) \int_{-\infty}^{\infty} d v^{\prime} \Psi\left(v^{\prime 2}+\tau^{2}\right)\right. \\
& \left.-\Psi\left(v^{2}+\tau^{2}\right) \int_{-\infty}^{\infty} d v^{\prime} \hat{D}\left(\tau, v^{\prime}, \theta\right)\right\}
\end{aligned}
$$


Equation (9) is quite formidable and appears to require approximate techniques. Assume a solution of the form

$$
\hat{D}=\Psi\left(v^{2}+\tau^{2}\right) e^{-i \Delta Q_{x} \theta} \sum_{n, m} a_{n, m} f_{n, m}(\tau, v),
$$

where the $f_{n, m}(\tau, v)$ 's are orthogonal basis functions with the weighting function $\Psi\left(v^{2}+\tau^{2}\right)$, the $a_{n, m}$ 's are constants, and $\Delta Q_{x}$ is the betatron tune shift. Insert Eq. (10) into Eq. (9), multiply by the complex conjugate $f_{p, q}^{*}(\tau, v) d \tau d v$, and integrate. This results in an eigenvalue equation for the $a_{n, m}$ 's

$$
\Delta Q_{x} a_{p, q}=\sum_{n, m} T_{p, q, n, m} a_{n, m} .
$$

There are two functional forms of $\Psi$ for which the matrix elements have a simple form. When $\Psi\left(v^{2}+\tau^{2}\right)=$ $\left(1-v^{2}-\tau^{2}\right)^{-1 / 2}$ with $v^{2}+\tau^{2}<1$, the line density $\rho(\tau)$ is a boxcar function. The expansion functions are

$$
\begin{aligned}
f_{k, m}(z)= & C_{k, m}[\tau+i \operatorname{sgn}(m) v]^{|m|} \\
& \times P_{k}^{-1 / 2,|m|}\left[2\left(v^{2}+\tau^{2}\right)-1\right],
\end{aligned}
$$

where

$$
\begin{aligned}
P_{k}^{-1 / 2, m}(x)= & \frac{1}{2^{k}} \sum_{p=0}^{k}\left(\begin{array}{c}
k-1 / 2 \\
p
\end{array}\right)\left(\begin{array}{l}
k+m \\
k-p
\end{array}\right) \\
& \times(x-1)^{k-p}(x+1)^{p}
\end{aligned}
$$

is a Jacobi polynomial [12], and

$$
C_{k, m}^{2}=\frac{\Gamma(k+1) \Gamma(|m|+k+1 / 2)(|m|+2 k+1 / 2)}{\pi \Gamma(k+1 / 2) \Gamma(|m|+k+1)} .
$$

For the boxcar line density, the discussion will be restricted to a wake potential of the form $W(\tau)=W=$ const. For this simple wake potential, the matrix element is given by

$$
\begin{aligned}
T_{p, q, n, m}= & \left(q Q_{s}-\Delta Q_{s c}\right) \delta_{q}^{m} \delta_{p}^{n}+\Delta Q_{s c} B_{n}^{m} B_{p}^{q} \frac{2 \delta_{|m|+2 n}^{|q|+2 p}}{2(|m|+2 n)+1} \\
& -\Delta Q_{W} \frac{B_{n}^{m} B_{p}^{q}}{2(|m|+2 n)+1}\left\{\delta_{|q|+2 p}^{0} \delta_{|m|+2 n}^{0}+\frac{+\delta_{|q|+2 p}^{|m|+2 n+1}-\delta_{|q|+2 p}^{|m|+2 n-1}}{2(|q|+2 p)+1}\right\},
\end{aligned}
$$

where $\Delta Q_{s c}>0$ is the incoherent tune depression in the beam, $-\Delta Q_{W}<0$ is the tune shift of the rigid mode due to the wake, and

$$
B_{n}^{m}=\left\{\frac{\Gamma(n+1 / 2)(2|m|+1+4 n) \Gamma(|m|+n+1 / 2)}{\pi \Gamma(n+1) \Gamma(|m|+n+1)}\right\}^{1 / 2} .
$$

When $\Delta Q_{W}=0$, the subspaces with $|m|+2 n=$ const are independent. For a given value of $|m|+2 n$, there are a finite number of equations and very accurate numerical solutions can be obtained.

When $\Psi\left(\tau^{2}+v^{2}\right)=\exp \left[-\left(\tau^{2}+v^{2}\right)\right]$, the expansion functions are $f_{k, m}=H_{k}(\tau) H_{m}(v)$, where $H_{k}(x)$ is the Hermite polynomial. For the same constant wake potential, the matrix element is

$$
\begin{aligned}
T_{p, q, n, m}= & -i Q_{s}\left\{n \delta_{m+1}^{q} \delta_{n-1}^{p}-m \delta_{m-1}^{q} \delta_{n+1}^{p}\right\} \\
& -\Delta Q_{s c} \frac{\delta_{m}^{q}\left[1-\delta_{m}^{0}\right]}{p ! \sqrt{2 \pi}}(-2)^{(n-p) / 2} \Gamma\left(\frac{n+p+1}{2}\right) \operatorname{even}(n+p) \\
& -\frac{2 \Delta Q_{W}}{\pi} \frac{\delta_{m}^{0} \delta_{q}^{0}}{p !}\left\{\delta_{p}^{0} \delta_{n}^{0} \pi / 2+\left(\delta_{p}^{0} \delta_{n}^{0}-1\right) \frac{(-2)^{(n-p-1) / 2}}{\sqrt{2}} \Gamma\left(\frac{n+p}{2}\right) \operatorname{even}(n+p+1)\right\} .
\end{aligned}
$$

In Eq. (13), $\Delta Q_{s c}$ is the peak value of the space charge tune depression in the bunch, and even $(n)=1$ if $n$ is even and is zero otherwise.

For a more general wake potential, one sets $\Delta Q_{W}=0$ in Eq. (13) and adds the term

$$
\delta T_{p, q, n, m}=\frac{-\delta_{m}^{0} \delta_{q}^{0} i^{n-p}}{4 Q_{x} 2^{p} p !} \int_{-\infty}^{\infty} d x x^{n+p} e^{-x^{2} / 2} \tilde{W}(x) .
$$

In Eq. (14),

$$
\tilde{W}(x)=\int_{0}^{\infty} W(\tau) e^{i x \tau} d \tau
$$

is the Fourier transform of the wake potential. In more physical variables,

$$
\tilde{W}(x)=\frac{i c q I_{b} Z_{\perp}\left(x / \sigma_{t} \sqrt{2}\right)}{\pi E_{T} \sigma_{t} \sqrt{2}},
$$

where $q$ is the charge per particle, $I_{b}$ is the bunch current, $Z_{\perp}(\omega)$ is the transverse impedance, $\sigma_{t}$ is the rms bunch duration, and $E_{T}$ is the total energy per particle.

While the matrix elements given by Eqs. (12) and (13) have closed forms, we know of no exact solutions to Eq. (11) when $\Delta Q_{W}$ and $Q_{s}$ are not zero.

To proceed, the matrix is truncated and solved numerically. With matrix element (12), the matrix is truncated by summing over $|m|+2 n \leq m_{\max }$, where $m_{\max }$ is the highest synchrotron mode included in the calculation. With matrix element (13), the matrix is truncated by summing over $m+n \leq m_{\max }$. Figure 1 shows the 


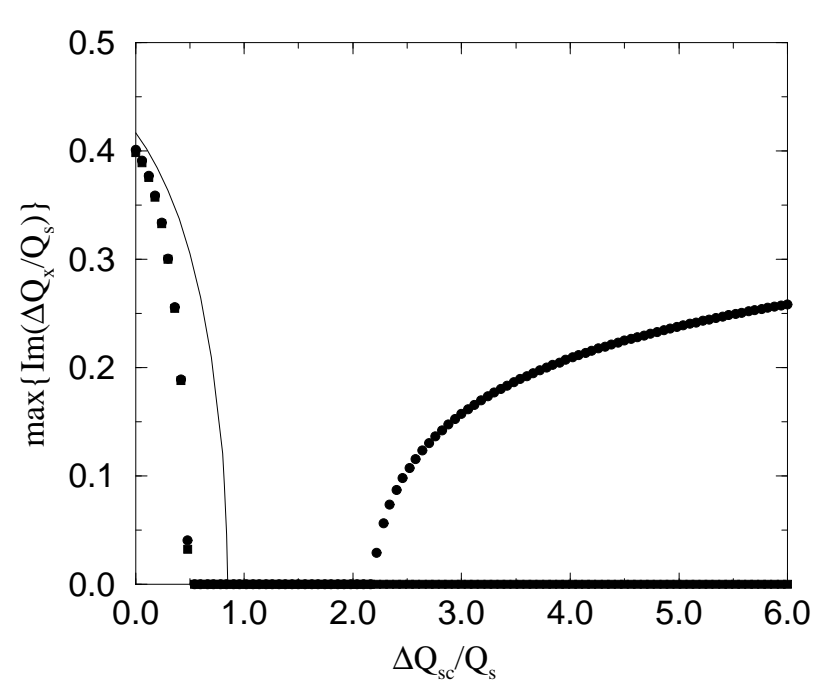

FIG. 1. Largest value of $\operatorname{Im}\left(\Delta Q_{x} / Q_{s}\right)$ as a function of $\Delta Q_{s c} / Q_{s}$ and $m_{\max }$ using matrix element (12). The value of $W$ is twice the size needed to produce instability with $\Delta Q_{s c}=0$ : $m_{\max }=1$, solid line; $m_{\max }=5$, squares; $m_{\max }=10$, circles.

growth rate of the most unstable mode as a function of $\Delta Q_{s c} / Q_{s}$ for the boxcar line density. The value of $W$ was twice that needed to produce instability with $\Delta Q_{s c}=0$. The behavior is not monotonic and suggests that there are ranges of space charge tune shift which improve stability. Next, consider the Gaussian distribution. Figure 2 shows growth rate versus $\Delta Q_{s c}$ for a simple wake that is $50 \%$ larger than the $\Delta Q_{s c}=0$ threshold. The growth rates for large tune shift are small compared to Fig. 1, but they would be dangerous in a real machine. Things are even less clear for other forms of impedance. Figure 3 shows the growth rates for a line density $\propto \exp \left(-\tau^{2}\right)$ when the

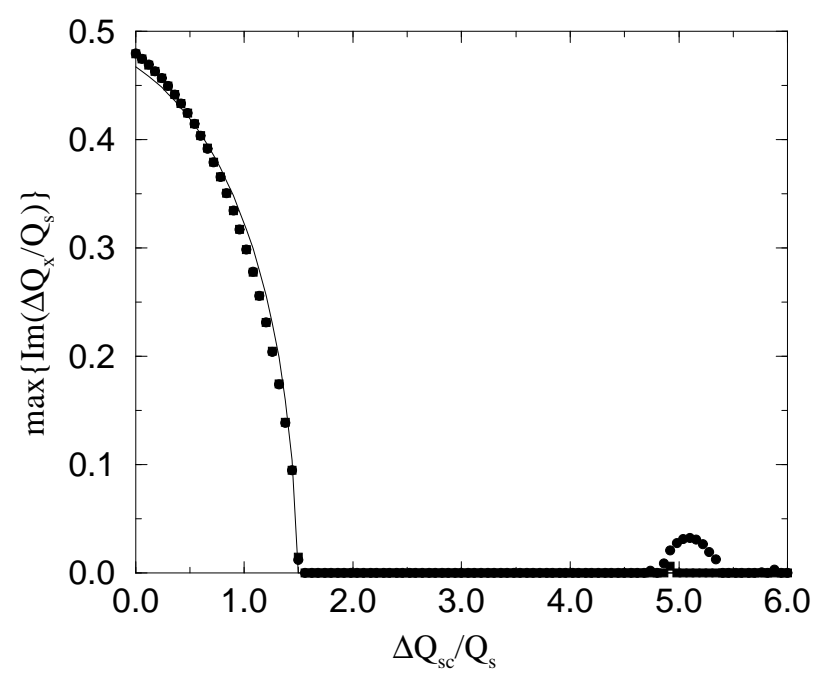

FIG. 2. Largest value of $\operatorname{Im}\left(\Delta Q_{x} / Q_{s}\right)$ as a function of $\Delta Q_{s c} / Q_{s}$ and $m_{\max }$ for a Gaussian density and simple wake. The wake is $50 \%$ larger than that needed to produce instability for $\Delta Q_{s c}=0: m_{\max }=1$, solid line; $m_{\max }=5$, squares; $m_{\max }=10$, circles. wake potential is of the form $W(\tau)=W_{0} \sin (2 \tau) \exp (-\tau)$ with $W_{0} 50 \%$ greater than that needed to produce instability with $\Delta Q_{s c}=0$. For this case, it is not clear that the expansion converges at all.

Some insight into the unreliability of the expansion technique can be obtained by considering the case $Q_{s}=0$ with the simple wake [13]. In this case, Eq. (9) can be integrated over $v$ yielding

$$
\frac{\partial \hat{D}(\tau, \theta)}{\partial \theta}=\frac{i W}{2 Q_{x}} \rho(\tau) \int_{-T}^{\tau} d \tau^{\prime} \hat{D}\left(\tau^{\prime}, \theta\right),
$$

where $\hat{D}(\tau, \theta)=\int d v \hat{D}(\tau, v, \theta)$. Set $\quad \hat{D}(\tau, \theta)=$ $x(\tau, \theta) \rho(\tau)$, and let

$$
u=\int_{-T}^{\tau} \rho\left(\tau^{\prime}\right) d \tau^{\prime}
$$

In these variables,

$$
\frac{\partial x(u, \theta)}{\partial \theta}=\frac{i W}{2 Q_{x}} \int_{0}^{u} d u^{\prime} x\left(u^{\prime}, \theta\right) .
$$

Consider the initial value problem and let $x(u, 0)=u^{k}$, where $k$ is an integer. Assuming a solution of the form

$$
x(u, \theta)=u^{k} \sum_{n=0}^{\infty} u^{n} f_{n}(\theta)
$$

yields

$$
x(u, \theta)=u^{k} k ! \frac{I_{k}\left(\sqrt{2 i W u \theta / Q_{x}}\right)}{\left(\sqrt{i W u \theta / 2 Q_{x}}\right)^{k}},
$$

where $I_{k}$ is the modified Bessel function of the first kind. For arguments with large real values, $I_{k}(x) \approx$ $\exp (x) / \sqrt{2 \pi x}$. Since the polynomials $u^{k}$ form a basis, the solution for arbitrary initial conditions satisfies $|x|=O\left[\exp \left(\sqrt{W u \theta / Q_{x}}\right)\right]$ as $\theta \rightarrow \infty$. This is a growing solution, but its growth rate, defined as $\operatorname{Im}\left(Q_{x}\right)$, is zero.

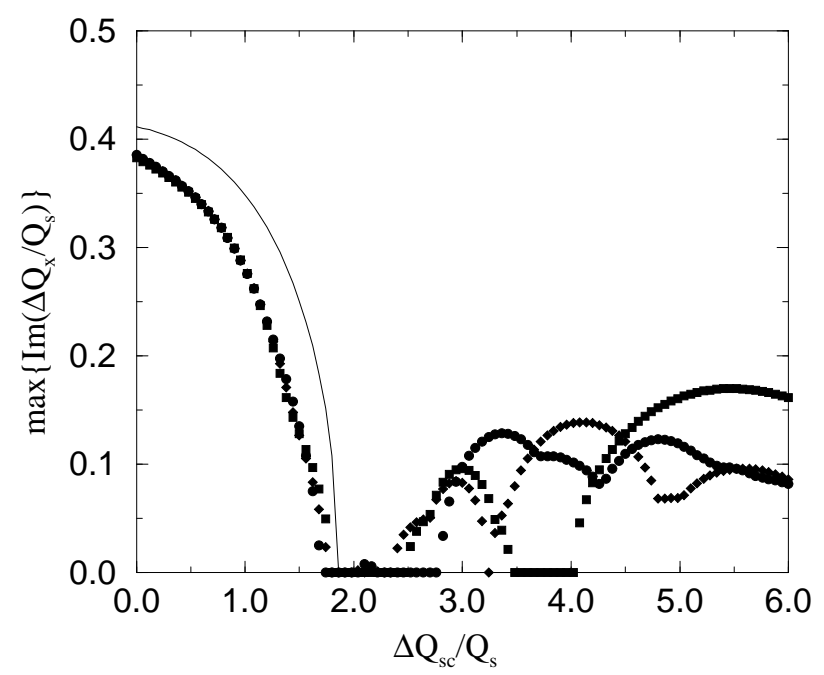

FIG. 3. Largest value of $\operatorname{Im}\left(\Delta Q_{x} / Q_{s}\right)$ as a function of $\Delta Q_{s c} / Q_{s}$ and $m_{\max }$ for the resonator impedance described in the text: $m_{\max }=1$, solid line; $m_{\max }=5$, squares; $m_{\max }=10$, circles; $m_{\max }=15$, diamonds. 
Conversely, consider the Gaussian distribution with $Q_{s} \rightarrow 0$ in $T_{p, q, n, m}$. This yields an eigenvalue problem which approximates Eq. (15). The growth rates are independent of $\Delta Q_{s c}$ and, as shown in Fig. 4, the peak growth rate drops slowly as $m_{\max }$ increases. While the growth rate as a function of $m_{\max }$ may converge to zero, it is difficult to tell. When taken in conjunction with the data presented in Figs. 1-4, it appears that the expansion technique does not always converge as more basis vectors are added, and this technique may not converge to the true answer when it does converge. In the next section, a technique which does not have these deficiencies is presented.

\section{SOLUTION VIA PARTICLE TRACKING}

An alternate approach to the problem is to solve it via particle tracking [7]. The bunch is modeled as $N$ interacting macroparticles. The equations of motion for the $k$ th macroparticle are taken to be

$$
\begin{gathered}
\frac{d^{2} \tau_{k}}{d^{2} \theta}=-Q_{s}^{2} \tau_{k} \\
\frac{d^{2} x_{k}}{d^{2} \theta}=-Q_{x}^{2} x_{k}+\frac{C_{s c}}{N} \sum_{j=1}^{N}\left(x_{k}-x_{j}\right) \lambda\left(\tau_{k}-\tau_{j}\right) \\
+\frac{1}{N} \sum_{j=1}^{N} x_{j} \hat{W}\left(\tau_{k}-\tau_{j}\right)
\end{gathered}
$$

In Eq. (19), the new functions $\lambda(\tau)$ and $\hat{W}(\tau)$ are introduced to smooth out the particle-particle forces. The individual macroparticles can be viewed as rigid narrow cylinders with the long axis along $\tau$. When the transverse offset between two such macroparticles is small compared

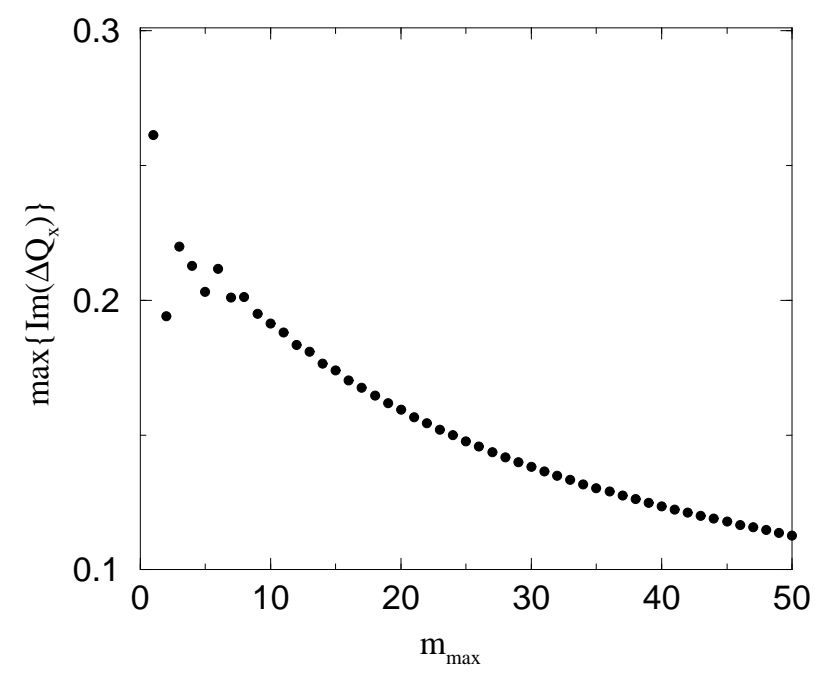

FIG. 4. Largest value of $\operatorname{Im}\left(\Delta Q_{x}\right)$ versus $m_{\max }$ for $Q_{s}=0$.

to the transverse size of the charge distribution, the transverse force varies almost linearly with displacement. If the initial transverse offsets are small compared to the cylinder radius and the system is stable, the transverse offsets are always small, at least in an average sense, and the approximation can be justified. Mathematically, if one takes the limit $N \rightarrow \infty$ and then takes the limits of $\lambda(\tau)$ going to a delta function and $\hat{W}(\tau)$ going to the wake potential, Eq. (2) is recovered.

The number of macroparticles is controlled using the parameter $n_{\ell}$. The initial longitudinal variables are selected by considering the subset of lattice points $\left(T_{k}, V_{k}\right)=\left[\left(k_{\tau}+1 / 2\right) / n_{\ell},\left(k_{v}+1 / 2\right) / n_{\ell}\right]$, with $k_{\tau}$ and $k_{v}$ integers, which are inside the unit circle. The initial longitudinal coordinates of a macroparticle $\left(\tau_{k}, v_{k}\right)$ are derived via

$$
\left(\tau_{k}, v_{k}\right)=\left(T_{k}, V_{k}\right) L\left[1-\left(1-T_{k}^{2}-V_{k}^{2}\right)^{1 /(1+\mu)}\right]^{1 / 2} /\left(T_{k}^{2}+V_{k}^{2}\right)^{1 / 2},
$$

where the parameter $\mu$ determines the bunch shape and $L$ is the half length of the bunch. The smoothed density in longitudinal phase space is proportional to $\left(L^{2}-\tau^{2}-\right.$ $\left.v^{2}\right)^{\mu}$, and results in a line density $\propto\left(L^{2}-\tau^{2}\right)^{\mu+1 / 2}$. In most cases, the longitudinal variables were updated once per turn with a rotation of angle $2 \pi Q_{s}$. Test cases where the rotation was performed more frequently were done to check that the results were essentially the same.

The initial values of the transverse variables $x$ and $p$ were obtained using a random number generator. The transverse dynamics consists of two parts, single particle dynamics and multiparticle dynamics. The scheme involves a single particle update followed by a multiparticle update and is repeated $M$ times per turn. The single particle update is given by a transfer matrix with a bare betatron phase advance $2 \pi Q_{x} / M$. The multiparticle update consists of a kick from the space charge and wake forces. The space charge kick on the $k$ th particle is

$$
F_{k}=\hat{C}_{s c} \sum_{j=1}^{N}\left(x_{k}-x_{j}\right) \lambda\left(\tau_{k}-\tau_{j}\right),
$$

where $\hat{C}_{s c}=2 \pi C_{s c} / N M$. The kick due to the wake potential is

$$
F_{k}=\frac{2 \pi}{N M} \sum_{j=1}^{N} x_{j} \hat{W}\left(\tau_{k}-\tau_{j}\right) .
$$

On the surface, Eqs. (20) and (21) appear to require $O\left(N^{2}\right)$ operations to obtain the kicks for all $N$ macroparticles, which would make simulations with large $N$ untenable. For appropriate choices of $\lambda(\tau)$ and $\hat{W}(\tau)$, the operation count drops to $O[N \log (N)]$. The trick is to 
generalize the phasor technique which is usually employed to retain the cumulative effects of multiple passages through a resonant structure [13].

Consider Eq. (20) with $\lambda(\tau)=\exp (-\alpha|\tau|)$, where $\int \lambda d t=2 / \alpha=\tau_{e}$ is the equivalent duration of $\lambda(\tau)$. Sort the values of $\tau_{j}$ so that $\tau_{j} \leq \tau_{j+1}$, which is an $O(N \log N)$ process with standard algorithms [14]. Equation (20) is given by

$$
F_{k} / \hat{C}_{s c}=x_{k} S 1_{k}^{-}-S 2_{k}^{-}+x_{k} S 1_{k}^{+}-S 2_{k}^{+},
$$

where

$$
\begin{aligned}
& S 1_{k}^{-}=\sum_{j=1}^{k} e^{\alpha\left(\tau_{j}-\tau_{k}\right)}, \\
& S 2_{k}^{-}=\sum_{j=1}^{k} x_{j} e^{\alpha\left(\tau_{j}-\tau_{k}\right)}, \\
& S 1_{k}^{+}=\sum_{j=k+1}^{N} e^{\alpha\left(\tau_{k}-\tau_{j}\right)}, \\
& S 2_{k}^{+}=\sum_{j=k+1}^{N} x_{j} e^{\alpha\left(\tau_{k}-\tau_{j}\right)}
\end{aligned}
$$

To calculate the sums, one starts with $S_{N}^{+}=0, S_{0}^{-}=0$ and uses

$$
\begin{aligned}
& S 1_{k+1}^{-}=e^{\alpha\left(\tau_{k}-\tau_{k+1}\right)} S 1_{k}^{-}+1, \\
& S 2_{k+1}^{-}=e^{\alpha\left(\tau_{k}-\tau_{k+1}\right)} S 2_{k}^{-}+x_{k+1}, \\
& S 1_{k-1}^{+}=e^{\alpha\left(\tau_{k-1}-\tau_{k}\right)}\left(S 1_{k}^{+}+1\right), \\
& S 2_{k-1}^{+}=e^{\alpha\left(\tau_{k-1}-\tau_{k}\right)}\left(S 2_{k}^{+}+x_{k}\right),
\end{aligned}
$$

Note that these recurrence relations are stable and that the kicks for all $N$ particles require $O(N)$ calculations after the particles have been sorted in arrival time. The sorting procedure is done when the $\tau_{k}$ 's are updated.

Next, consider the kick due to the transverse wakefield. Set

$$
\hat{W}(\tau)=C_{W} \int_{-\infty}^{\tau} \lambda\left(\tau^{\prime}\right) d \tau^{\prime},
$$

where $C_{W}$ is a constant and $\lambda(t)=\exp (-\alpha|\tau|)$, as before. Adjusting the constant so that $\hat{W}(\tau) \rightarrow W$ as $\tau \rightarrow \infty$ yields

$$
F_{k}=\frac{2 \pi W}{N M}\left[S 0_{k}^{-}-S 2_{k}^{-} / 2+S 2_{k}^{+} / 2\right],
$$

where

$$
S 0_{k}^{-}=\sum_{j=1}^{k} x_{j} .
$$

For a resonator impedance, the wake kick on particle $k$ is given by

$$
F_{k}=\sum_{j=1}^{k} x_{j} W_{0} \sin \left[\tilde{\omega}\left(\tau_{k}-\tau_{j}\right)\right] e^{-\omega_{r}\left(\tau_{k}-\tau_{j}\right) / 2 Q},
$$

where $\omega_{r}$ is the resonant frequency, $Q$ is the quality factor, and $\tilde{\omega}=\omega_{r} \sqrt{1-1 / 4 Q^{2}}$. Since $\sin (\tilde{\omega} \tau) \exp \left(-\omega_{r} \tau / 2 Q\right)=\exp \left(i \tilde{\omega} \tau-\omega_{r} \tau / 2 Q\right) / 2 i-$ $\exp \left(-i \tilde{\omega} \tau-\omega_{r} \tau / 2 Q\right) / 2 i$, the wake kick can be expressed as two sums similar to $S 2^{-}$.

The choice $\lambda(\tau)=\exp (-\alpha|\tau|)$ is somewhat disconcerting. This can be seen from the fact that $\lambda(\tau)$ is proportional to the autocorrelation function of the current pulse due to a single macroparticle. For $\lambda(\tau)=$ $\exp (-\alpha|\tau|)$, the current pulse for a single macroparticle is $\propto K_{0}(|\alpha \tau|)$, a modified Bessel function which has a logarithmic singularity at $\tau=0$. A less singular current pulse is associated with the smoothing function $\lambda(\tau)=(1+|\alpha \tau|) \exp (-\alpha|\tau|)$, which has an equivalent width $\tau_{e}=4 / \alpha$. For this smoothing function, the current pulse for a single macroparticle is $\propto \exp (-\alpha|\tau|)$, which is continuous. The same kind of techniques used with $\lambda(\tau)=\exp (-\alpha|\tau|)$ can be applied to the smoother function although the expressions are more complicated. The space charge kick is given by

$$
\begin{aligned}
F_{k} / \hat{C}_{s c}= & x_{k}\left(1+\alpha \tau_{k}\right) S 1_{k}^{-}-\left(1+\alpha \tau_{k}\right) S 2_{k}^{-} \\
& -x_{k} \alpha S 3_{k}^{-}+\alpha S 4_{k}^{-}+x_{k}\left(1-\alpha \tau_{k}\right) S 1_{k}^{+} \\
& -\left(1-\alpha \tau_{k}\right) S 2_{k}^{+}+x_{k} \alpha S 3_{k}^{+}-\alpha S 4_{k}^{+},
\end{aligned}
$$

with

$$
\begin{aligned}
& S 3_{k+1}^{-}=e^{\alpha\left(\tau_{k}-\tau_{k+1}\right)} S 3_{k}^{-}+\tau_{k+1}, \\
& S 4_{k+1}^{-}=e^{\alpha\left(\tau_{k}-\tau_{k+1}\right)} S 4_{k}^{-}+x_{k+1} \tau_{k+1}, \\
& S 3_{k-1}^{+}=e^{\alpha\left(\tau_{k-1}-\tau_{k}\right)}\left(S 3_{k}^{+}+\tau_{k}\right), \\
& S 4_{k-1}^{+}=e^{\alpha\left(\tau_{k-1}-\tau_{k}\right)}\left(S 4_{k}^{+}+x_{k} \tau_{k}\right) .
\end{aligned}
$$

The wake kick is given by

$$
\begin{gathered}
F_{k}=\frac{2 \pi W}{N M}\left[S 0_{k}^{-}-\left(2-\alpha t_{k}\right) S 2_{k}^{-} / 4-\alpha S 4_{k}^{-} / 4\right. \\
\left.+\left(2-\alpha t_{k}\right) S 2_{k}^{+}+\alpha S 4_{k}^{+} / 4\right] .
\end{gathered}
$$

The space charge kick obtained using either Eq. (22) or (28) is an exact pairwise sum over the macroparticles in the beam and satisfies

$$
F_{k}=\frac{\partial}{\partial x_{k}} U\left(x_{1}, x_{2}, \ldots, x_{N}, \tau_{1}, \tau_{2}, \ldots, \tau_{N}\right),
$$

where

$$
U=\frac{\hat{C}_{s c}}{4} \sum_{j, k}\left(x_{j}-x_{k}\right)^{2} \lambda\left(\tau_{j}-\tau_{k}\right) .
$$

Therefore, the update map for the bare rotation followed by a space charge kick is symplectic. Since the wake force is causal, there is no symplectic update scheme that represents it. However, the direct pairwise sum should be at least as good as any other technique.

Since Eq. (19) is linear in the transverse variables, any exponentially growing solution will continue to grow without saturating. This makes it possible to obtain accurate numbers for the growth rate of the most unstable mode. To measure the growth rate of the most unstable mode, the bunch was divided into 10 bins in $\tau$, where each 
bin contained the same number of macroparticles. The average values of $x$ and $p$ were calculated for each bin and the Courant-Snyder (CS) invariants were obtained. Figure 5 shows the $\log$ of the CS invariants for these bins versus the number of turns for no space charge and a simple wake at twice the head-tail threshold. For clarity, the initial variables were $x=1, p=0$. The other input parameters were $n_{\ell}=50, \mu=1, Q_{x}=2.925, M=24$, $\tau_{e}=0.02$, and $Q_{s}=0.01$.

For the simple case illustrated in Fig. 5, an unambiguous measurement of the growth rate is made with a short simulation. Different parameters can lead to growth rates which are more difficult to obtain. Figure 6 shows the CS invariants for $Q_{s}=0$; other are parameters the same as in Fig. 5. All of the particles were started with $x=1$, $p=0$. From Eq. (17) one expects that the CS invariants should grow as $\log (|\mathrm{CS}|) \approx 2 \log \left(A_{0}\left|I_{0}(\sqrt{i \alpha\langle u\rangle \theta})\right|\right)$, where the average is taken over a longitudinal bin. The parameters $A_{0}$ and $\alpha$ were obtained by a simultaneous least squares fit to the 10 curves in Fig. 6 using the Marquardt algorithm. For the best fit parameters, the root mean square difference between the data and the fit was $\left\langle(\text { data }- \text { fit })^{2}\right\rangle^{1 / 2}=0.038$, which is small compared to the variation in the data, $\sim 20$. The best fit parameters were $A_{0}=1.012$ and $\alpha=0.0887$. From the simulation input parameters, the analytic calculation gives $A_{0}=1$ and $\alpha=0.0894$.

The update algorithm includes five physical parameters $Q_{x}, L, Q_{s}, \Delta Q_{s c}, W_{0}$, and $\mu$, and three numerical parameters $\tau_{e}, M$, and $n_{\ell}$. For the step function wake, the bunch length and the equivalent width of the smoothing function reduce to a single parameter $\tau_{e} / L$. This leaves an eight-dimensional parameter space. A uniform grid search of this space is impossible, so the philosophy was to choose a set of physical parameters and then find a

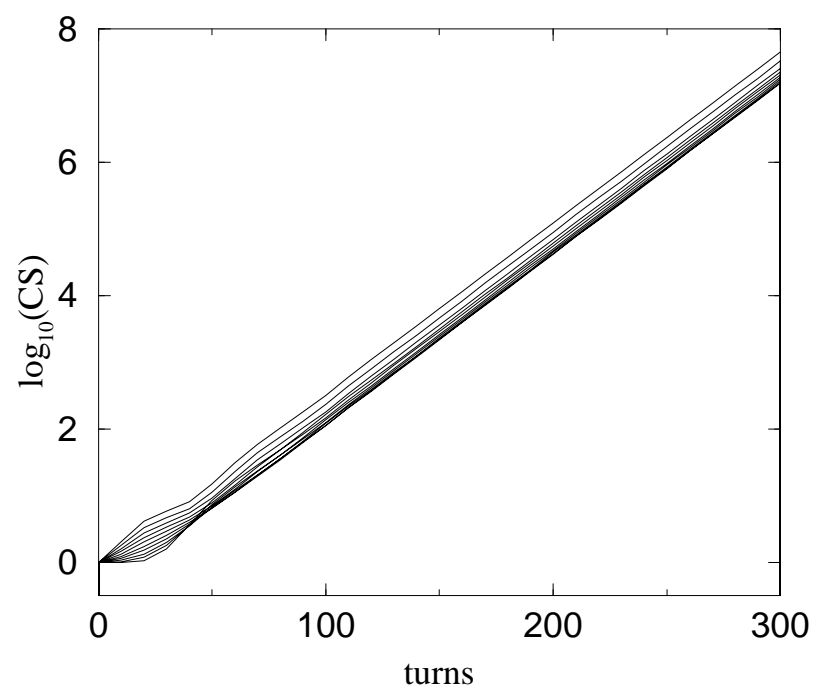

FIG. 5. Log of CS invariants versus turn number for a system at twice the head-tail threshold with no space charge and $Q_{s}=0.01$. region of the numerical parameters in which the physical results, such as growth rate and mode shape, did not change.

Figure 7 shows growth rate versus $\tau_{e} / L$ for $\mu=1$, $\Delta Q_{s c}=0.2, \quad Q_{s}=0.01, \quad Q_{x}=2.925, \quad W_{0}=2$, and a variety of numerical parameters. For $\Delta Q_{s c}=0$, the instability threshold is $W_{0}=0.45$. For the case shown, $\Delta Q_{s c} / Q_{s}=20$ and $\operatorname{Im}\left(Q_{x} / Q_{s}\right) \lesssim 0.01$ for $0.03 \leq \tau_{e} / L \leq 0.05$. It is worthwhile to note that for $\tau_{e} / L=0.02$ and $n_{\ell}=50$ (solid line), there are $\sim 100$ macroparticles in a longitudinal slice of length $\tau_{e}$. Even for this fairly high particle density, it appears that numerical simulations have not converged. Note that Fig. 2 in [7] is at odds with Fig. 7. This is due to the fact that the simulations used to obtain the earlier figure were too short. The simulations used here were run for $10^{5}$ turns.

Given the convergence problems evident in Fig. 7, most of the numerical results were obtained for smaller $\Delta Q_{s c}$. The convergence rates were substantially faster and the numerical results are reasonably certain. Figure 8 shows growth rates versus $\Delta Q_{s c} / Q_{s}$ with other parameters the same as in Fig. 7. It is essentially the same as Fig. 3 in [7], with the exception that the lower limit on the vertical axis is more appropriate here. Consider the secondary bump in the growth rates. The rates for $n_{\ell}=$ 100 are smaller than the results for $n_{\ell}=50$, which in turn are mostly less than the results for $n_{\ell}=25$. It seems reasonable to assume that the secondary bump is a numerical artifact and that the threshold space charge tune shift satisfies $\Delta Q_{s c} / Q_{s} \approx 2$. Simulations like this were performed for a variety of parameters and instability thresholds as a function of $\Delta Q_{s c} / Q_{s}$ were obtained. In all cases with $Q_{s} \neq 0$, an unstable system showed exponential growth. Slow growth, as in Eq. (17), was eventually dominated by exponential growth or was absent.

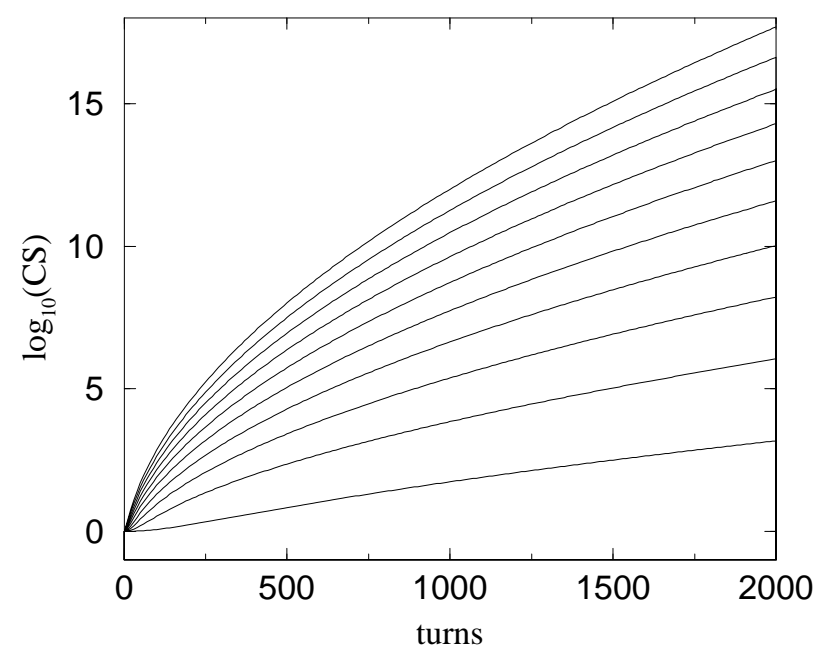

FIG. 6. Log of CS invariants versus turn number for the same conditions as in Fig. 5, except that $Q_{s}=0$. 


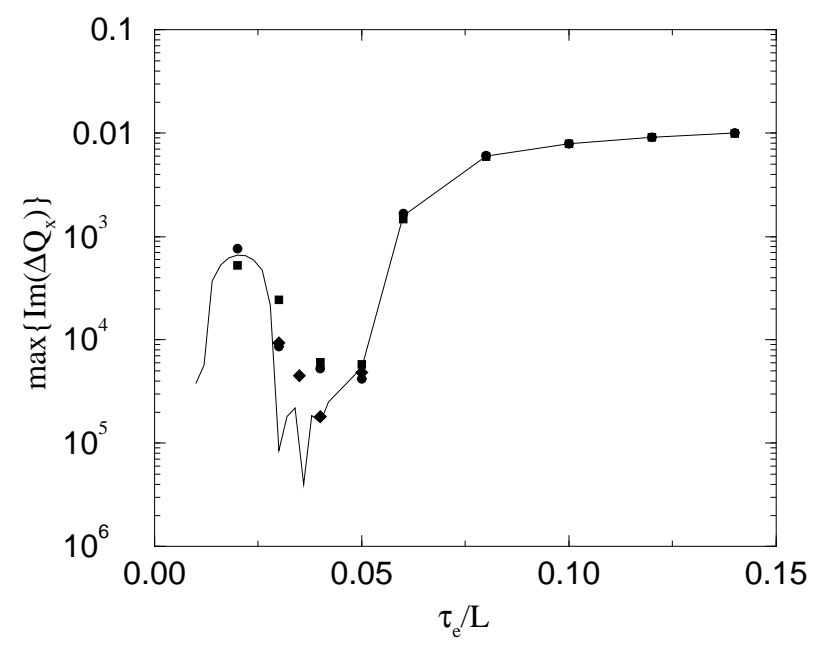

FIG. 7. $\operatorname{Im}\left(\Delta Q_{x}\right)$ vs $\tau_{e} / L$ with physical parameters $\mu=1$, $\Delta Q_{s c}=0.2, Q_{s}=0.01, Q_{x}=2.925, W_{0}=2$. The numerical parameters are $n_{\ell}=25, M=12$, circles; $n_{\ell}=25, M=24$, squares; $n_{\ell}=50, M=24$, solid line; $n_{\ell}=100, M=24$, diamonds.

The results are summarized in Fig. 9. Even with the precautions taken, the results for $\Delta Q_{s c} / Q_{s}>5$ are suspect. Nonetheless, it appears that incoherent space charge forces increase the fast head-tail threshold. It is natural to ask what the underlying scaling laws are and how other factors, such as chromaticity, change the picture. Unfortunately, the computational requirements are daunting. It is particularly worrisome when one considers the parameter regime in which planned and existing machines operate. Fortunately, there is a solvable model which agrees with the computational results in regimes where the computations are trustworthy.

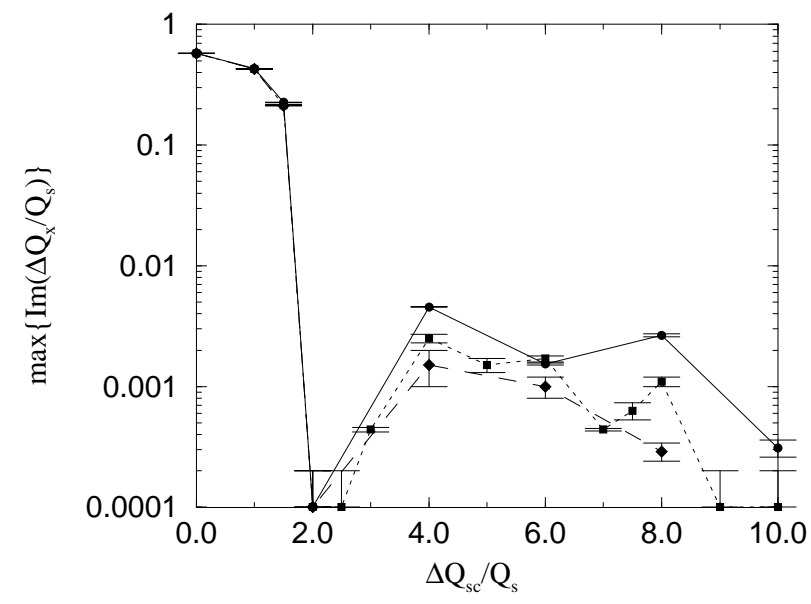

FIG. 8. Largest value of $\operatorname{Im}\left(\Delta Q_{x} / Q_{s}\right)$ vs $\Delta Q_{s c} / Q_{s}$ with $L=$ $\mu=1$ for various values of $n_{\ell}$ and $\tau_{e}: n_{\ell}=25, \tau_{e}=0.05$, circles, solid line; $n_{\ell}=50, \tau_{e}=0.02$, squares, short dashed line; $n_{\ell}=100, \tau_{e}=0.01$, diamonds, long dashed line. The value of the step function wake was 2.5 times larger than the threshold value with $\Delta Q_{s c}=0$.

\section{SQUARE WELL MODEL}

The first step to obtain this model is to modify the longitudinal dynamics. Generalize Eq. (1) to read

$$
\frac{d^{2} \tau}{d \theta^{2}}=\frac{v}{d \theta}=-\frac{d U(\tau)}{d \tau}
$$

Model the longitudinal potential using a square well

$$
U(\tau)= \begin{cases}0, & \text { if } 0<\tau<\tau_{b} \\ U_{0}, & \text { otherwise }\end{cases}
$$

Take the air bag [13] longitudinal phase space distribution $\Psi(\tau, v) \propto \delta\left(v-v_{0}\right)+\delta\left(v+v_{0}\right)$ for $0 \leq \tau \leq$ $\tau_{b}$, where $\tau_{b}$ is the full bunch length. The line density is a boxcar function and there is a well-defined synchrotron tune $Q_{s}=\pi v_{0} / \tau_{b}$.

Before presenting the analytic results, consider simulations using the square well. These are compared with the quadratic potential and a boxcar line density $(\mu=-1 / 2)$ in Fig. 10. Both simulations used the same number of macroparticles, identical smoothing lengths, etc. As is clear from the figure, the growth rates and thresholds are very similar for the two cases. Growth rates versus space charge tune shift are shown in Fig. 11. As with Fig. 10, only the phase space distribution and longitudinal forces are different for the two cases. As is clear from the figures, an air bag distribution in a square well and a box car distribution with a quadratic potential have very similar behavior, especially when compared with typical error bars on machine impedances. The air bag distribution is now considered in detail.

Let $X_{+}(\theta, \tau)$ be the transverse offset of particles with $d \tau / d \theta=-v_{0}$ (positive momentum offset below transition) at azimuth $\theta$ and arrival time $\tau$. Define

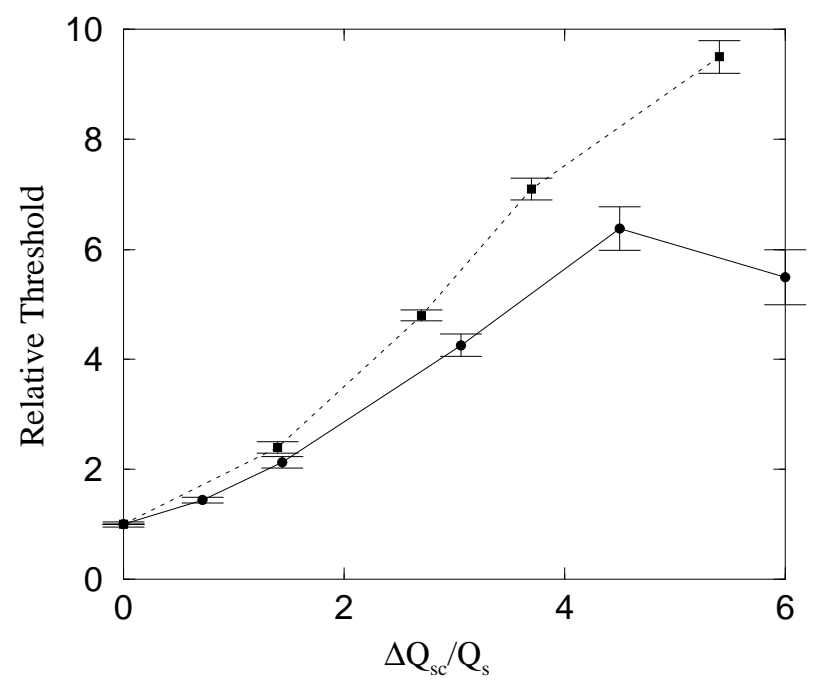

FIG. 9. Threshold wake in units of the threshold wake for $\Delta Q_{s c}=0$ vs $\Delta Q_{s c} / Q_{s}$ for different line densities: $\mu=1$, circles, solid line; $\mu=-1 / 2$, squares, short dashed line. 


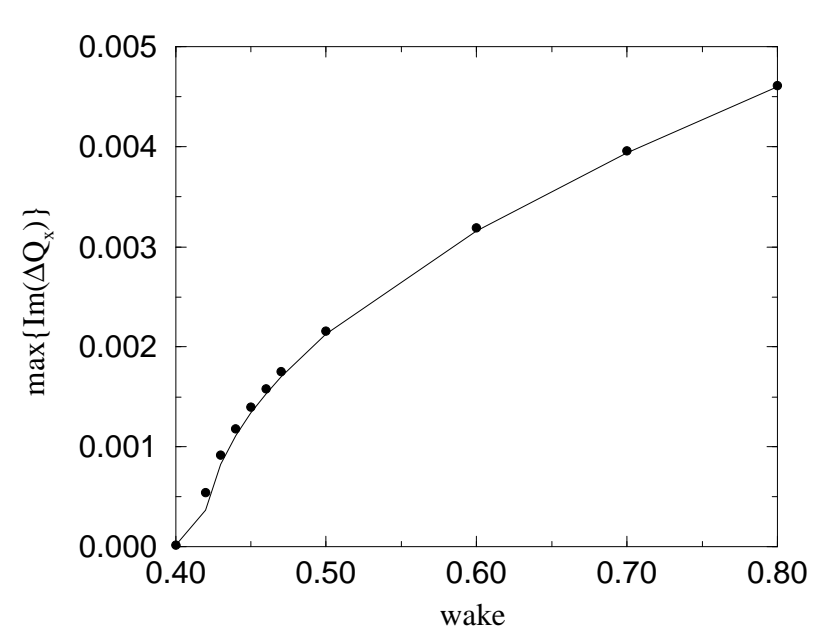

FIG. 10. Largest value of $\operatorname{Im}\left(\Delta Q_{x}\right)$ versus wake strength from simulations with $\Delta Q_{s c}=0, Q_{s}=0.01$ and different longitudinal forces: $\mu=-1 / 2$ in a quadratic potential, circles; square well model, solid line. For wake $<0.4$ there was no instability.

$X_{-}(\theta, \tau)$ in similar fashion for particles with $d \tau / d \theta=$ $+v_{0}$. Assume that all betatron tune shifts are small compared to the betatron tune so that

$$
X_{+}(\theta, \tau)=e^{-i Q_{x} \theta} x_{+}(\theta, \tau),
$$

where the variation of $x_{+}(\theta, \tau)$ is slow in $\theta$, and similarly for $X_{-}$. Then

$$
\begin{aligned}
\frac{d x_{+}}{d \theta}= & \frac{\partial x_{+}}{\partial \theta}-v_{0} \frac{\partial x_{+}}{\partial \tau} \\
= & i \Delta Q_{s c}\left\{x_{+}(\theta, \tau)-\bar{x}(\theta, \tau)\right\}-i \xi v_{0} x_{+} \\
& +i \kappa \int_{0}^{\tau} d s W(\tau-s) \bar{x}(\theta, s) \\
& + \text { contributions from previous turns }
\end{aligned}
$$

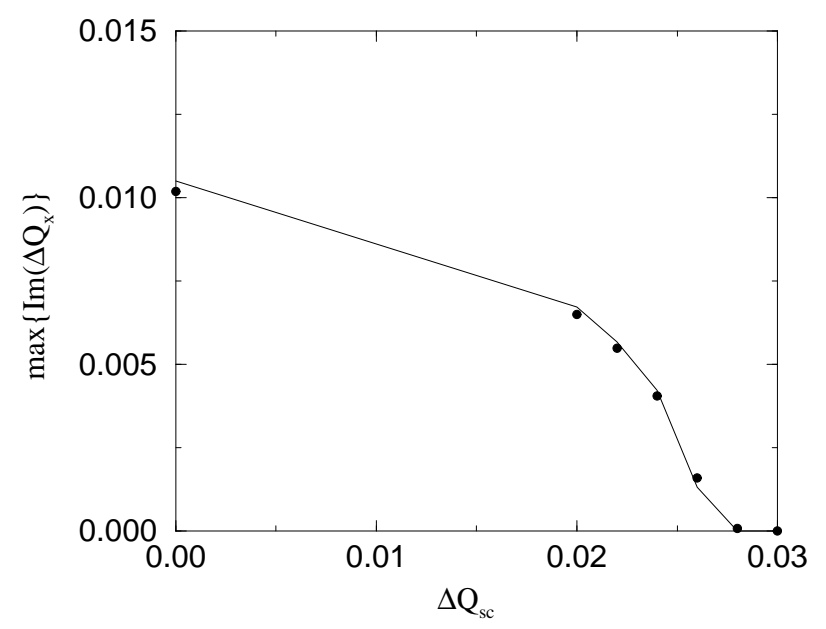

FIG. 11. Largest value of $\operatorname{Im}\left(\Delta Q_{x}\right)$ vs $\Delta Q_{s c}$ from simulations with $Q_{s}=0.01$ and different longitudinal forces: $\mu=-1 / 2$ in a quadratic potential, circles; square well model, solid line.

$$
\begin{aligned}
\frac{d x_{-}}{d \theta}= & \frac{\partial x_{-}}{\partial \theta}+v_{0} \frac{\partial x_{-}}{\partial \tau} \\
= & i \Delta Q_{s c}\left\{x_{-}(\theta, \tau)-\bar{x}(\theta, \tau)\right\}+i \xi v_{0} x_{-} \\
& +i \kappa \int_{0}^{\tau} d s W(\tau-s) \bar{x}(\theta, s) \\
& + \text { contributions from previous turns }
\end{aligned}
$$

In Eqs. (31) and (32), $\bar{x}=\left(x_{+}+x_{-}\right) / 2$ is the transverse centroid of the beam. Remembering that $\tau=\omega_{0} t-\theta$,

$$
\kappa=\frac{I_{0} c}{4 \pi \beta\left(E_{T} / q\right) \omega_{0}^{2} Q_{x}},
$$

where $q$ is the particle charge, $E_{T}=\gamma m c^{2}$ is the total particle energy, $\beta=v / c$, and $I_{0}$ is the peak current. Consider the eigenvalue problem where $\partial / \partial \theta \rightarrow-i \Delta Q_{x}$ so that the coherent betatron tune is $Q_{x}+\Delta Q_{x}$. Assume the wake potential is given by $W(\tau)=W_{0} \exp (-\alpha \tau)$, which is easily generalized to any impedance which can be represented as a sum of poles. The equations become

$$
\begin{aligned}
\frac{d x_{+}(\tau)}{d \tau}= & -i\left\{\frac{\Delta Q_{s c} / 2+\Delta Q_{x}-\xi v_{0}}{v_{0}}\right\} x_{+} \\
& -i F / v_{0}+i\left(\Delta Q_{s c} / 2 v_{0}\right) x_{-}, \\
\frac{d x_{-}(\tau)}{d \tau}= & +i\left\{\frac{\Delta Q_{s c} / 2+\Delta Q_{x}+\xi v_{0}}{v_{0}}\right\} x_{-} \\
& +i F / v_{0}-i\left(\Delta Q_{s c} / 2 v_{0}\right) x_{+}, \\
\frac{d F(\tau)}{d \tau}= & -\alpha F+\left(\kappa W_{0} / 2\right)\left\{x_{-}+x_{+}\right\} .
\end{aligned}
$$

In these units the head-tail phase shift is $\chi=-\xi \tau_{b} / 2$. The boundary conditions are $x_{+}(0)=x_{-}(0)$ and $x_{+}\left(\tau_{b}\right)=x_{-}\left(\tau_{b}\right)$, since an instantaneous change in $v$ leaves $x$ and $p_{x}$ unchanged. Also, the wake force is continuous. For a single bunch in the machine,

$$
F(0)=e^{-2 \pi i\left(Q_{x}+\Delta Q_{x}\right)-\left(2 \pi-\tau_{b}\right) \alpha} F\left(\tau_{b}\right) .
$$

For more complex impedances, such as a resonator, the force is written as a sum of terms. Each term obeys an equation such as (36), where $\alpha$ is complex, and each obeys (37). These generalizations will not be done here. Additionally, this text always refers to a single bunch in the ring. The extension of these computations to $N_{b}$ identical, symmetrically filled bunches is done by substituting $Q_{x} \rightarrow\left(Q_{x}+s\right) / N_{b}$, where $0 \leq s<N_{b}$ is the coupled bunch mode number, and choosing the units of $\tau$ to be radians of azimuth between bunch centers. The coupling between bunches can be ignored by setting $F(0)=0$, which must be done for a consistent picture when $\alpha=0$. The results presented in this paper use $F(0)=0$ to concentrate on mode coupling instabilities. 
Before considering the general case, there are some analytic results. When $W_{0}=0$, the eigenfunctions are given by $x_{ \pm, k}=\exp (i \xi \tau)\left\{\cos \left(k \pi \tau / \tau_{b}\right) \mp\right.$ $\left.\left(i \tau_{b} \Delta Q_{x} / k \pi v_{0}\right) \sin \left(k \pi \tau / \tau_{b}\right)\right\}$, where $k$ is an integer. The tune shifts are given by $\Delta Q_{x}=$ $-\Delta Q_{s c} / 2 \pm \sqrt{\left(\Delta Q_{s c} / 2\right)^{2}+\left(k Q_{s}\right)^{2}}$, where the + is used for $k \geq 0$. The eigenfunctions form a complete set and the tunes are real so the system is stable. Note that the tune shift for the $k=0$ mode vanishes because $\Delta Q_{x}$ measures the difference between the coherent tune and the low current tune.

When $\alpha \tau_{b} \gg 1$, the wake force is given by

$$
\begin{aligned}
F & =\kappa \int_{0}^{\tau} d s W(\tau-s) \bar{x}(\theta, s) \approx \kappa \int_{0}^{\infty} d s W(s) \bar{x}(\tau, \tau-s) \\
& \approx \kappa \int_{0}^{\infty} d s W(s)\left\{\bar{x}(\theta, \tau)-s \frac{d \bar{x}(\theta, \tau)}{d \tau}\right\} \approx \frac{\kappa W_{0}}{\alpha}\left\{\bar{x}(\theta, \tau)-\frac{1}{\alpha} \frac{d \bar{x}(\theta, \tau)}{d \tau}\right\} .
\end{aligned}
$$

When the last expression is used in Eqs. (34) and (35), the problem is reasonably straightforward. The analog of the $k=0$ space charge mode has a tune shift $\Delta Q_{x}=-G(1-i \xi / \alpha)$, where $G=\kappa W_{0} / \alpha$. The modes for $k \neq 0$ have

$$
\Delta Q_{x}=-\Delta Q_{s c}-\frac{G(1-i \xi / \alpha)-\Delta Q_{s c} \pm \sqrt{\left[G(1-i \xi / \alpha)-\Delta Q_{s c}\right]^{2}+4 k^{2} Q_{s}^{2}\left(1-G^{2} / 4 \alpha^{2} v_{0}^{2}\right)}}{2-G^{2} / 2 \alpha^{2} v_{0}^{2}} .
$$

The system is stable only if $G<2 \alpha v_{0}$ and space charge plays no role. This result is similar to that found by Zotter for a Gaussian longitudinal distribution using the basis expansion technique [15].

For the general case, notice that the differential equations are of the form

$$
\frac{d \mathbf{U}}{d \tau}=\mathbf{M U},
$$

where $\mathbf{M}$ is a constant matrix and, except for special cases,

$$
\mathbf{U}=\sum_{k=1}^{3} \mathbf{U}_{k} e^{\lambda_{k} \tau}
$$

The $\mathbf{U}_{k}$ 's and $\lambda_{k}$ 's can be obtained to machine precision with standard routines. The computational strategy is to choose a starting value for $\Delta Q_{x}$ and iterate until the

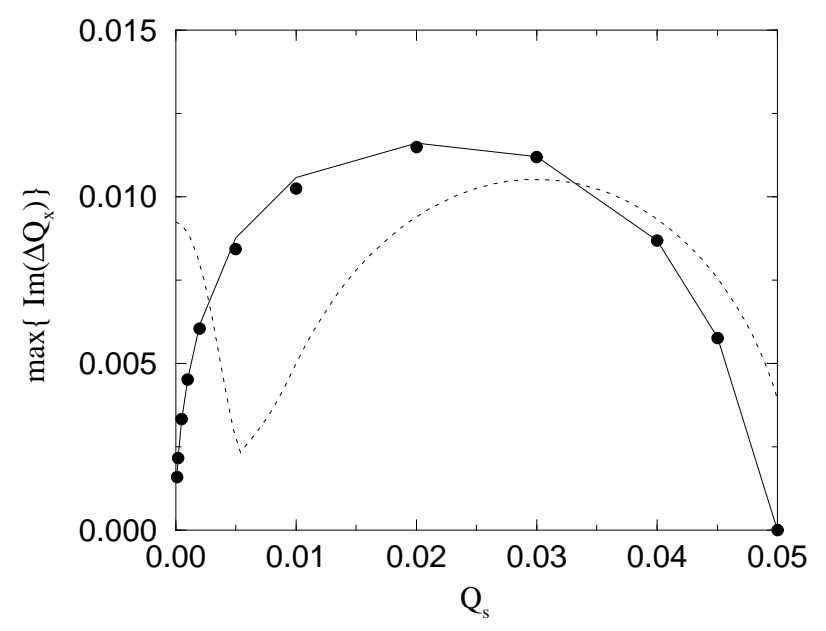

FIG. 12. Largest value of $\operatorname{Im}\left(\Delta Q_{x}\right)$ vs $Q_{s}$ for $\Delta Q_{s c}=$ $\alpha=\xi=0$ with $\kappa W_{0} \tau_{b}=0.055$ : simulations in a harmonic potential with $\mu=-1 / 2$ circles; analytic square well model, solid line; basis expansion for the boxcar distribution with $m_{\max }=5$, short dashed line. value of $\Delta Q_{x}$ corresponds to a solution that satisfies the boundary conditions. By choosing a range of starting values for $\Delta Q_{x}$, one can be confident that the most unstable mode has been found.

Figure 12 shows the growth rate of the most unstable mode with fixed wake strength and no coupling between bunches as a function of $Q_{s}$ for the three kinds of calculations. The air bag distribution in a square well and the simulations in a quadratic potential are the same within 5\%, while the basis expansion technique is clearly different. Figure 13 shows the growth rate versus the space charge tune shift for $\tau_{b}=\kappa=Q_{s}=1$ and $\xi=$ $\alpha=0$. For $\Delta Q_{s c}=0$, the threshold value of the wake strength is $W_{0}=1.15$. For $\alpha>0$, the trend remains. Figure 14 shows the threshold value of $W_{0}$ versus space charge tune shift for the same bunch parameters and various values of $\alpha$ with $\chi=0$. Since Eqs. (34)-(36)

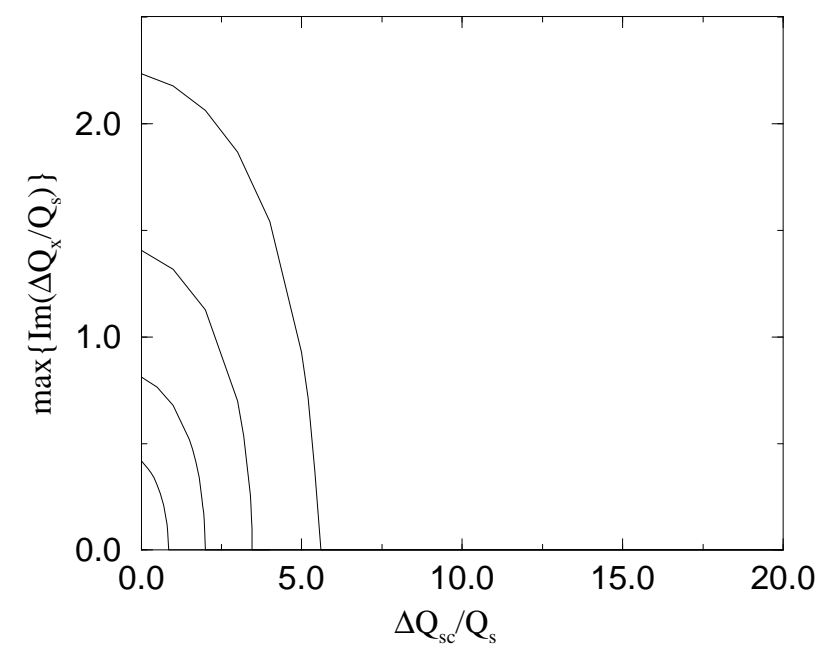

FIG. 13. Largest value of $\operatorname{Im}\left(\Delta Q_{x} / Q_{s}\right)$ vs $\Delta Q_{s c} / Q_{s}$ for the analytic square well model with $W_{0}=2,4,8,16$ and $\alpha=\xi=$ 0 . For $\Delta Q_{s c}=0, W_{0}=1.16$ at threshold. 


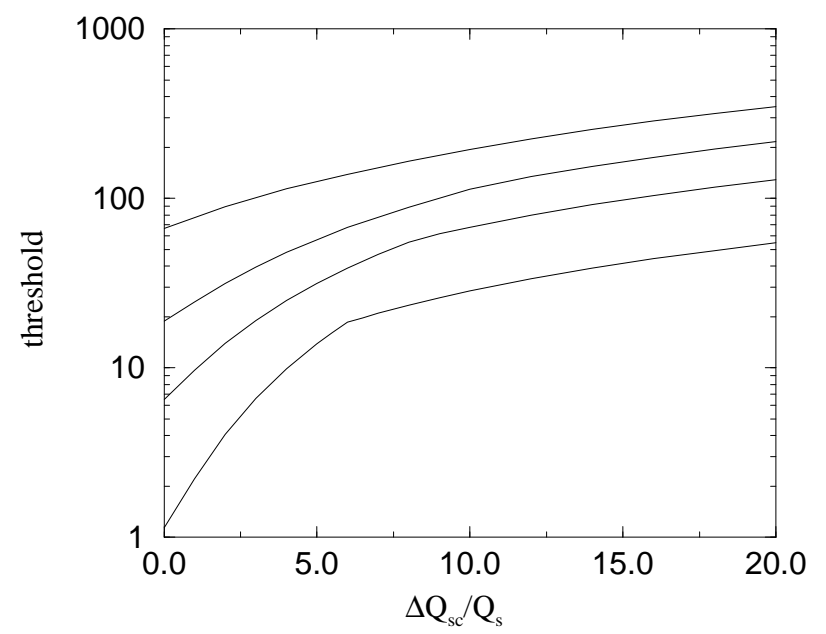

FIG. 14. Threshold value of $\kappa W_{0} \tau_{b} / Q_{s}$ vs $\Delta Q_{s c} / Q_{s}$ for the analytic square well with $\chi=0$ and $\alpha \tau_{b}=0,5,10,20$, from bottom to top.

with $F(0)=0$ imply

$$
\frac{\Delta Q_{x}}{Q_{s}}=\frac{\Delta Q_{x}}{Q_{s}}\left(\chi, \alpha \tau_{b}, \frac{\Delta Q_{s c}}{Q_{s}}, \frac{\kappa W_{0} \tau_{b}}{Q_{s}}\right),
$$

the curves in Fig. 14 are generally applicable. For $\Delta Q_{s c}=0$ and $\alpha \tau_{b} \gg 1$, the threshold wake satisfies $\kappa W_{0} \approx 0.5 * v_{0} \alpha^{2}$, which is a factor of 4 less than the analytic result. The threshold increases with space charge tune shift. For $\Delta Q_{s c} / Q_{s}>10$, the curves in Fig. 14 are nearly straight lines;

$$
\left\{\frac{\kappa W_{0} \tau_{b}}{Q_{s}}\right\}_{\text {thresh }}=a\left(\alpha \tau_{b}\right)+b\left(\alpha \tau_{b}\right) \frac{\Delta Q_{s c}}{Q_{s}} .
$$

Using a least square fit, the coefficients are $a(0)=2.16$, $b(0)=2.63 ; a(5)=5.48, b(5)=6.18 ; a(10)=11.80$, $b(10)=10.22$; and $a(20)=37.4, b(20)=15.6$.

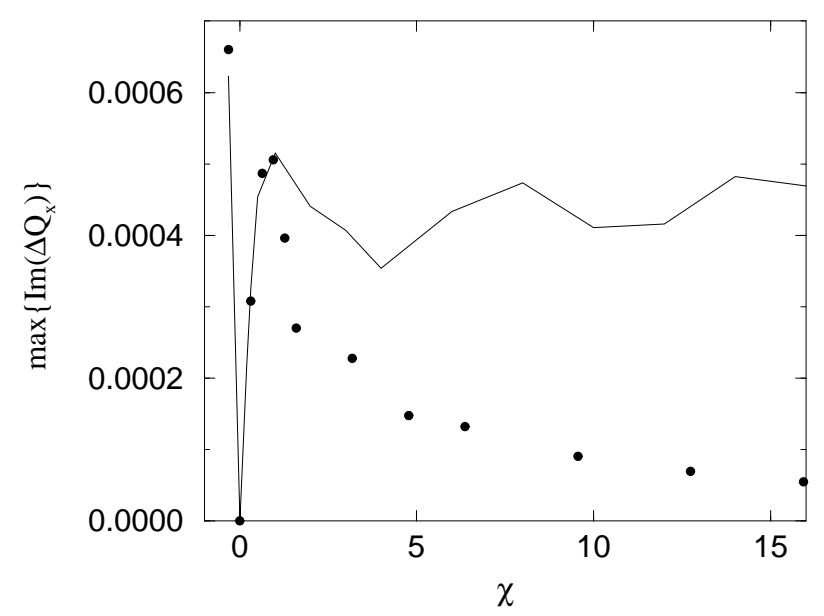

FIG. 15. Largest value of $\operatorname{Im}\left(\Delta Q_{x}\right)$ vs $\chi$ for $\Delta Q_{s c}=0$, $Q_{s}=0.01$ with $\alpha=0$ and $W_{0}$ at half the threshold for the fast head-tail instability; analytic square well model, solid line; $\mu=-1 / 2$ in a quadratic potential, circles.
The square well model encounters difficulty when chromaticity is included. As is clear from Fig. 15, tracking in a quadratic potential and the square well model agree for small head-tail phase shifts, but the tracking growth rates decrease for large values of head-tail phase while the growth rates from the square well model remain large. Conversely, if the square well model predicts a sufficiently stable system, the actual system should be sufficiently stable.

\section{CONCLUSIONS}

The square well model of transverse bunched beam stability agrees with simulations in some parameter regimes where the simulations can be trusted. Both models predict that space charge should strongly damp the transverse mode coupling instability. The importance of bunch length relative to the range of the wakefield was verified. If the range of the wakefield is short, the beam can be stable even if the tune shift is larger than the synchrotron tune. Hadron bunches tend to be long with their transverse impedance dominated by space charge, and both effects suppress the fast head-tail instability. From a computational point of view, the square well model is faster and more robust than the other techniques, but it can overestimate growth rates for nonzero chromaticity. One suspects that this derives from its sparse momentum distribution. This conjecture is testable, in principle, since the model can be generalized to any longitudinal potential which is piecewise constant.

\section{ACKNOWLEDGEMENTS}

Thanks to Thomas Roser and Francesco Ruggiero for careful reading of the manuscript and useful comments. This work was supported by the United States Department of Energy under Contract No. DE-AC02-98CH10886.

[1] T.P. R. Linnecar and E. N. Shaposhnikova, in Fourth European Particle Accelerator Conference, London, 1994 (World Scientific, Singapore, 1994), p. 1093.

[2] G. Besnier, D. Brandt, and B. Zotter, Part. Accel. 17, 51 77 (1985).

[3] M. Blaskiewicz and W. T. Weng, Phys. Rev. E 50, 4030 (1994).

[4] F. Sacherer, CERN Report No. 77-13, 1977.

[5] Y. Chin, K. Satoh, and K. Yokoya, Part. Accel. 13, 45 (1983).

[6] K. Satoh and Y. Chin, Nucl. Instrum. Methods Phys. Res. 207, 309 (1983).

[7] M. Blaskiewicz, 1997 Particle Accelerator Conference, Vancouver, Canada (to be published).

[8] A. G. Ruggiero, in BNL Report No. 51236, 1979, p. 91.

[9] K. J. Kim, in BNL Report No. 51236, 1979, p. 100.

[10] R. Baartman, in Proceedings of the International Workshop on Particle Dynamics in Accelerators, Tsukuba, 
Japan, 1994 (KEK, Ibaraki, Japan, 1995), p. 273, and references therein.

[11] F. Ruggerio, Report No. CERN-LEP-TH/84-21, 1984.

[12] I. S. Gradshteyn and I. M. Ryzhik, Table of Integrals Series and Products (Academic, New York, 1965).

[13] A.W. Chao, Physics of Collective Beam Instabilities in
High Energy Accelerators (Wiley, New York, 1993), p. 214.

[14] See, e.g., W. H. Press, B.P. Flannery, S. A. Teukolsky, and W.T. Vetterling, Numerical Recipes (Cambridge University, Cambridge, 1986).

[15] B. Zotter, Report No. CERN/ISR-TH/82-10, 1982. 